\title{
SAÚDE DO TRABALHADOR: INTOXICAÇÃO POR AGROTÓXICOS NO BRASIL
}

\author{
Vinicius Cassu Queiroz ; Rebecca Piscitello dos Santos ${ }^{2}$; Mateus Dias Antunes ${ }^{3}$; \\ Marcelo Picinin Bernuci ${ }^{4}$; Ely Mitie Massuda ${ }^{5}$ \\ ${ }^{1}$ Acadêmico do curso de Medicina do Centro Universitário de Maringá - \\ UNICESUMAR - Maringá, Paraná, Brasil. \\ ${ }^{2}$ Acadêmica do curso de Medicina do Centro Universitário de Maringá - \\ UNICESUMAR - Maringá, Paraná, Brasil. \\ ${ }^{3}$ Mestrando em Promoção da Saúde do Centro Universitário de Maringá - \\ UNICESUMAR - Maringá, Paraná, Brasil. Bolsista CAPES. \\ ${ }^{3}$ Professores Doutores do Programa de Pós-Graduação em Promoção da Saúde do \\ Centro Universitário de Maringá - UNICESUMAR - Maringá, Paraná, Brasil e \\ Pesquisadores do Instituto Cesumar de Ciência,Tecnologia e Inovação - ICETI \\ elymitie@hotmail.com
}

Recebido em: 08/04/2017 - Aprovado em: 10/06/2017 - Publicado em: 20/06/2017 DOI: 10.18677/EnciBio_2017A135

\begin{abstract}
RESUMO
O Brasil ocupa posição de destaque na produção mundial de alimentos, mas também lidera o ranking no consumo de agrotóxicos. Com o objetivo de avaliar a produção científica sobre intoxicação por agrotóxicos de trabalhadores rurais, realizou-se um estudo cienciométrico da literatura nacional nas bases de dados da Literatura Latino-americana e Caribe em Ciências da Saúde, Medical Literature Analysis and Retrieval System On line - Medline por meio da Biblioteca Virtual em Saúde, e Scientific Eletronic Library Oonline - Scielo. A quase totalidade da literatura científica brasileira sobre o tema é realizada por instituições públicas, principalmente das regiões Nordeste e Sul e publicadas em periódicos exclusivamente do CentroSul brasileiro. Poucos dados epidemiológicos devido à intoxicação em trabalhadores e na população de modo geral são observados. O número total de trabalhos encontrados sobre o tema é relativamente baixo, dada a dimensão do setor na economia brasileira.
\end{abstract}

PALAVRAS-CHAVE: Agrotóxicos; Saúde da população rural; Promoção da saúde.

\section{RURAL WORKER HEALTH: INTOXICATION BY AGROCHEMICALS IN BRAZIL}

\begin{abstract}
Brazil occupies a prominent position in the world food production, but also leads the ranking in the consumption of agrochemicals. With the objective of evaluating the scientific production on pesticide intoxication of rural workers, a scientific study of the national literature was carried out in the databases of the Latin American and Caribbean Literature in Health Sciences, Medical Literature Analysis and Retrieval System Online - Medline through the Virtual Health Library, and Scientific Eletronic Library Oonline - Scielo. Almost all Brazilian scientific literature on the subject is carried out by public institutions, mainly from the Northeast and South regions and
\end{abstract}


published in periodicals exclusively from the Brazilian Center-South. Few epidemiological data due to intoxication in workers and in the general population are observed. The total number of works found on the subject is relatively low given the size of the sector in the Brazilian economy.

KEYWORDS: Pesticides; Health of the rural population; Health promotion.

\section{INTRODUÇÃO}

O Brasil, país que provê parte da demanda mundial de alimentos, é também conhecido como um dos maiores consumidores de agrotóxicos do planeta. Comparado a 1990, a produção agropecuária brasileira cresceu com base, principalmente, no aumento da produtividade: a produção agrícola brasileira de 2014 mais que dobrou em termos de volume e a produção pecuária triplicou (OCDE/FAO, 2016). Por outro lado, desde 2008, o Brasil é o maior consumidor mundial de agroquímicos. Entre 2002 e 2014 apresentou um crescimento de $190 \%$ na comercialização destes produtos (ABREU \& ALONZO, 2014).

O manuseio de produtos fitossanitários na atividade agrícola é uma prática antiga (ROCHA \& OLIVEIRA, 2016). Alguns registros se referem à utilização de sulfurados a partir do século XI, bem como de arsênios em 1700. Porém, no século $\mathrm{XX}$, com o emprego do DDT (diclorodifeniltricloroetano), foi comprovada a eficácia do controle químico na produtividade vegetal (TAVELLA et al., 2011). Desde então, com o início da "Revolução Verde", em 1950, a agricultura deixou de ter um aspecto familiar para converter-se em uma prática comercial. Desta forma, ocorreu a substituição de mão de obra pela maquinaria, o uso de agrotóxicos em larga escala e, por último, a introdução da biotecnologia (SILVA et al., 2005).

O início da utilização de agroquímicos na produção brasileira se deu por volta de 1960, associado a Programas de Saúde Pública, objetivando o combate a parasitas e vetores que representavam um sério problema de saúde pública (TAVELLA et al. 2011). Deste modo, o Brasil adentrava na Revolução Verde, programa lançado pelo governo que visava o aumento da produção agrícola com altos investimentos na fertilização do solo, mecanização, redução do custo de manejo e aplicação de defensivos agrícolas, os chamados agrotóxicos (PAPINI \& NAKAGAWA, 2014). A implantação deste Sistema trouxe avanços para a agropecuária nacional. No entanto, com o aumento da utilização dos agrotóxicos os problemas de saúde relacionados à manipulação e ingestão oral e respiratória desses produtos também se multiplicaram (MACHADO \& SANTOS, 2015).

$\mathrm{Na}$ América Latina, o Brasil é responsável por $86 \%$ do total empregado de agrotóxicos, consumo esse que se reflete diretamente sobre a saúde do trabalhador rural (FERREIRA \& VIANA JÚNIOR, 2015). O grande risco no manuseio desses produtos advém do uso incorreto, à acentuada nocividade de alguns, ao desrespeito às normas de segurança do trabalho e à falha da vigilância em saúde (MELLO \& SILVA, 2013).

Os fitossanitários podem causar intoxicação subaguda, aguda ou crônica (CARVALHO et al., 2017). A intoxicação subaguda se caracteriza pelo aparecimento dos sintomas de forma lenta, a depender do grau de exposição ao produto tóxico bem como ao seu tipo. Os sintomas podem ser subjetivos, como cefaleia, fraqueza, mal-estar e sonolência (GERAGE et al., 2017). Já na intoxicação aguda os sintomas aparecem abruptamente devido à exposição a produtos altamente tóxicos (FERREIRA \& OLIVEIRA, 2016). Por fim, a intoxicação crônica evidencia-se por um surgimento tardio, após um longo período de exposição de 
pequena a moderada intensidade, ocasionando danos irreversíveis à saúde, como paralisias e neoplasias (OPAS, 1997).

No que se refere à intoxicação por defensivos agrícolas, os grupos mais atingidos consistem em trabalhadores de baixa renda, produtores marginais ou assalariados (CARVALHO et al., 2017). Em se tratando do sexo, mulheres apresentam maior vulnerabilidade, uma vez que possuem não só maior gordura corporal, mas também, uma porcentagem maior de tecidos susceptíveis a hormônios (MELLO \& SILVA, 2013).

Há ainda casos de intoxicação por agrotóxicos devido à tentativa de suicídio, constituindo esta, a terceira causa de suicídio no Brasil (VIEIRA et al., 2015). Entre 1999 e 2009, foram notificadas cerca de 62.000 contaminações por agrotóxicos de uso agrícola, cerca de 5.600 por ano. Este mesmo sistema revela um total de 1.876 mortes no mesmo período, cerca de 170 ao ano (TEIXEIRA et al., 2014). Entretanto, há poucos dados epidemiológicos referentes à morbidade ou mortalidade por agrotóxicos na literatura. Essa ausência de informações refere-se mais comumente a países emergentes, onde a efetividade das normas de controle de comercialização e o manejo são duvidosos (BARBOSA, et al., 2014). Para cada caso notificado existem outros 50 não notificados (TEIXEIRA et al., 2014).

A conscientização e o conhecimento aprofundado a respeito dos malefícios destes elementos para a saúde do trabalhador rural e da população de modo geral é imprescindível para o país, já que este é considerado emergente, no qual há carência de informações e notificações a respeito do manejo e intoxicação por estes agroquímicos (BARBOSA et al., 2014).

Por fim, é relevante o levantamento a respeito da produção de conhecimento sobre o assunto para todos os profissionais da saúde, buscando-se criar incentivo para a formação destes, gerando profissionais para atuar no Sistema Único de Saúde (SUS). Tais profissionais devem estar habilitados para assistir a população, objetivando estratégias de intervenção, principalmente, no meio rural (SIQUEIRA \& KRUSE, 2008). O contexto atual de utilização intensiva e indiscriminada de agrotóxicos, somada ao manuseio incorreto pelos trabalhadores rurais, não traz boas perspectivas quanto a redução do número de intoxicações agudas ou agravos a saúde devido à exposição em longos períodos (ABREU \& ALONZO, 2014).

A importância do levantamento e sistematização das pesquisas relacionadas aos casos de intoxicação por agrotóxico no Brasil ressalta-se na medida em que o país é um dos grandes consumidores mundiais deste insumo agrícola. Desta forma, o objetivo da pesquisa consistiu na análise da evolução das publicações científicas sobre o problema da intoxicação de trabalhadores rurais e da população por agrotóxicos no Brasil, por meio da cienciometria.

\section{MATERIAL E MÉTODOS}

O presente trabalho consistiu em um estudo cienciométrico de literatura científica sobre a intoxicação por agrotóxicos no Brasil. De natureza quantitativa, a cienciometria possibilita conhecer a extensão e a abrangência das publicações sobre determinado tema. As recomendações metodológicas pautaram-se nas orientações de SCHUBERT et al. (1989) e BRAUN \& SCHUBERT (1988) para estudos cienciométricos.

O objeto de análise foi a produção científica veiculada em periódicos indexados nas bases eletrônicas de dados da Biblioteca Virtual em Saúde (BVS) disponível no site http://bvsalud.org, da Literatura Latino-Americana e do Caribe em 
Ciências da Saúde (LILACS), acessível no site http://lilacs.bvsalud.org, Medical Literature Analysis and Retrieval System On line (MEDLINE), acessível no site https://www.nlm.nih.gov/pubs/factsheets/medline.html\# e da Scientific Eletronic Library Online (SCIELO), disponível no site http://www.scielo.org/php/index.php, no período de 2010 a 2015.

Para a busca dos artigos científicos, realizada nos meses de fevereiro e março de 2016, valendo-se dos seguintes descritores: "agrotóxicos", "defensivo agrícola", "fitossanitários", "agroquímicos" e "intoxicação". O processo de busca, primeiramente, permitiu a identificação de 305 artigos científicos que foram, por sua vez, selecionados considerando-se a base de dados, o eixo temático central da pesquisa e o ano de publicação do artigo.

Desta forma, a partir da análise dos títulos e resumos, foram excluídos trabalhos cujos descritores não foram relacionados ao eixo central da pesquisa, ou seja, intoxicação. Após a avaliação, 17 trabalhos atenderam aos requisitos de seleção e a avaliação crítica possibilitou a identificação das seguintes categorias: local de realização da pesquisa; instituições participantes nas pesquisas; ano de publicação do artigo científico; unidade federativa de publicação e classificação do periódico segundo o critério Qualis Capes na área saúde coletiva de 2015. A análise quantitativa dos dados coletados foi realizada por meio da estatística descritiva, frequência simples e absoluta. Os dados foram tabulados e organizados em planilhas do programa Microsoft Excel 2013.

Sendo assim, foram assinalados os períodos de concentração das publicações sobre os aspectos destacados; elencadas as principais revistas científicas nacionais e internacionais que abordaram o tema; avaliadas as publicações coletadas considerando aspectos quantitativos e qualitativos. Os dados foram organizados e tabulados utilizando-se o programa Microsoft Excel 2010.

\section{RESULTADOS E DISCUSSÃO}

O número total de trabalhos encontrados sobre 0 assunto foi relativamente baixo, considerado o uso intensivo de agrotóxicos no Brasil e o número de notificações de intoxicações verificadas, sendo alguns deles revisões de literatura. $O$ fato não deixa de ser preocupante, pois o Brasil possui uma economia baseada no agronegócio, além de ser o maior consumidor mundial de defensivos agrícolas (ABREU \& ALONZO, 2014; FERREIRA \& VIANA JÚNIOR, 2015).

A tabela 1 apresenta a concentração temporal das publicações no período de 2010 a 2015. Dos 17 artigos selecionados, dois foram publicados em 2010 (11,76\%); três em 2011(17,65\%); um em 2012 (5,88\%); quatro em 2013 (23,53\%); quatro em 2014 (23,53\%); e três em 2015 (17,65\%).

TABELA 1. Número de publicações por ano.

\begin{tabular}{ccc}
\hline ANO DE PUBLICAÇÃO & N & $\%$ \\
\hline 2010 & 2 & 11,76 \\
2011 & 3 & 17,65 \\
2012 & 1 & 5,88 \\
2013 & 4 & 23,53 \\
2014 & 4 & 23,53 \\
2015 & 3 & 17,65 \\
2016 & - & - \\
\hline Total & 17 & 100 \\
\hline
\end{tabular}

Fonte: elaborado pelos autores 
A Tabela 2 elenca os periódicos que publicaram artigos sobre o tema e seus respectivos conceitos Qualis Capes em saúde coletiva. O Qualis Capes - Periódicos é um sistema de classificação de produção intelectual utilizado pela Capes (Coordenação de Aperfeiçoamento de Pessoal de Nível Superior), autarquia vinculada ao Ministério da Educação, para classificar as publicações de artigos científicos dos programas de pós-graduação. Os periódicos são avaliados por áreas e passam, anualmente, por atualizações. Os periódicos são categorizados em estratos por indicativos de qualidade sendo o A1, o mais elevado; A2; B1; B2; B3; B4; B5; C - com peso zero (CAPES, 2016).

TABELA 2. Principais periódicos de publicação.

\begin{tabular}{lccc}
\hline Periódicos & ISSN & QUALIS 2015* $^{*}$ & N \\
\hline Ciência e Saúde Coletiva & $1413-8123$ & B1 & 4 \\
Cadernos Saúde Coletiva (UFRJ) & $1414-462 X$ & B1 & 1 \\
Epidemiologia e Serviços de Saúde & $1679-4974$ & B2 & 3 \\
Interface - Comunicação, Saúde, Educação & $1424-3228$ & B1 & 1 \\
Revista Brasileira de Neurologia & $0101-8469$ & B4 & 1 \\
Revista Brasileira de Plantas Medicinais & $1516-0572$ & B2 & 1 \\
Brazilian Archives of Biology and Technology & $1516-8913$ & B2 & 1 \\
Revista de Saúde Pública & $0034-8910$ & A2 & 1 \\
Jornal Brasileiro de Nefrologia & $0101-2800$ & B2 & 1 \\
Revista Brasileira de Enfermagem & $0034-7167$ & B1 & 1 \\
Jornal Brasileiro de Psiquiatria (UFRJ) & $0047-2085$ & B2 & 1 \\
Revista Brasileira de Epidemiologia & $1415-790 X$ & B1 & 1 \\
\hline Total & & & 17 \\
\hline
\end{tabular}

Fonte: elaborado pelos autores * Classificação em Saúde Coletiva

Os 17 artigos selecionados foram publicados em 12 diferentes periódicos científicos. A revista Ciência e Saúde Coletiva apresentou o maior número de artigos sobre o tema, quatro ao todo, seguida pela revista Epidemiologia e Serviços de Saúde, com três publicações. Os demais periódicos publicaram um artigo no período considerado.

No que se refere ao Qualis Capes, segundo classificação na área de Saúde Coletiva, a Revista Saúde Pública apresentou o maior conceito, A2; Ciência e Saúde Coletiva, Cadernos Saúde Coletiva (UFRJ), Interface - Comunicação, Saúde, Educação, Revista Brasileira de Enfermagem e Revista Brasileira de Epidemiologia, conceito B1. Epidemiologia e Serviços de Saúde, Revista Brasileira de Plantas Medicinais, Brazilian Archives of Biology and Technology, Jornal Brasileiro de Nefrologia, além do Jornal Brasileiro de Psiquiatría (UFRJ) apresentaram conceito B2 e a Revista Brasileira de Neurologia, B4. Nenhum dos artigos foi publicado em periódicos Qualis Capes A1, B3, B5 ou C.

Segundo a tabela 3, a maior parcela das publicações ocorreu em periódicos do Estado do Rio de Janeiro, com um total de sete publicações, isto é, $41,8 \%$ do total. Seguiu-se o Estado de São Paulo, apresentando cinco publicações, ou seja, 29,41\%; o Distrito Federal, com 23,53\%; por fim, o Paraná com 5,88\% das publicações. Estes dados apontam para uma polarização das publicações envolvendo o tema, especialmente na região sudeste onde mais de $70 \%$ das publicações ocorreram. 
Assim, observa-se que todos os artigos selecionados sobre o tema foram publicados em periódicos das regiões sudeste, sul e no Distrito Federal.

TABELA 3. Número de publicações por unidade federativa

\begin{tabular}{lcc}
\hline Periódicos & $\mathbf{N}$ & $\%$ \\
\hline Rio de Janeiro & 7 & 41,18 \\
Distrito Federal & 4 & 23,53 \\
São Paulo & 5 & 29,41 \\
Paraná & 1 & 5,88 \\
\hline Total & 17 & 100 \\
\hline
\end{tabular}

Fonte: elaborado pelos autores

Os impactos biopsicossociais do uso de agrotóxicos não estão sendo devidamente mensurados e as condições de saúde e segurança do trabalhador rural brasileiro permanecem obscuras. Uma ocorrência relevante está no fato de estados brasileiros que fazem fronteira com países latinos, possuírem pouca ou nenhuma linha de pesquisa vinculada ao tema em função da facilidade de aquisição de agroquímicos contrabandeados, de qualidade duvidosa e normalmente mais baratos (SOARES \& PORTO, 2012).

Conforme Tabela 5, em se tratando da região de realização da pesquisa, observou-se uma heterogeneidade, já que quatro destas foram realizadas no nível nacional; duas no Centro-Oeste; cinco no Nordeste; uma na região Sudeste; três na região Sul e nenhuma na região Norte. Dois dos artigos analisados não fizeram referência ao local de realização da pesquisa.

TABELA 4. Número de artigos segundo a região de realização da pesquisa.

\begin{tabular}{lc}
\hline REGIÃO & N \\
\hline Brasil & 4 \\
Centro-Oeste & 2 \\
Nordeste & 5 \\
Norte & - \\
Sudeste & 1 \\
Sul & 3 \\
Local não mencionado & 2 \\
\hline Total & 17 \\
\hline
\end{tabular}

Fonte: elaborado pelos autores.

O maior número de pesquisas ocorreu na região Nordeste do Brasil, seguida pela região Sul. Em regiões com economia predominantemente advinda do agronegócio como Centro-Oeste e Norte realizaram apenas duas pesquisas publicadas sobre o tema. Essa incoerência demonstra que, mesmo que a maior parte das intoxicações por agrotóxicos ocorram em trabalhadores da área rural provenientes dessas regiões de agronegócio, aparentemente, há pouco interesse nesta linha de pesquisa nas regiões mencionadas anteriormente (SANTANA et al., 2013).

No que se refere ao número de instituições envolvidas na pesquisa que originaram as publicações, totalizaram 22, segundo a Tabela 5, cinco delas são Universidades Federais (22,73\%); seis (27,27\%), Universidades Estaduais; uma 
(4,54\%), Universidade Privada. Outras nove $(40,92 \%)$ foram instituições públicas que também participaram das pesquisas, como secretarias de saúde e institutos de pesquisas e uma organização internacional $(4,54 \%)$.

TABELA 5. Instituições participantes nas pesquisas sobre o tema intoxicações

\begin{tabular}{lcc}
\hline INSTITUIÇÕES & N & $\%$ \\
\hline Universidades Federais & 5 & 22,73 \\
Universidades e Faculdades Estaduais & 6 & 27,27 \\
Universidades Privadas & 1 & 4,54 \\
Instituições Públicas & 9 & 40,92 \\
Organizações Internacionais & 1 & 4,54 \\
\hline Total & 22 & 100
\end{tabular}

Fonte: elaborado pelos autores.

Os dados revelaram que a produção de conhecimento sobre o tema de forma quase absoluta, são de financiamento e iniciativa pública, como secretarias de saúde, institutos de pesquisas e universidades. Nenhuma pesquisa ou trabalho no período foi financiado ou tem pesquisadores vinculados a indústrias de agroquímicos. $O$ fato condiz com a Lei oㅜ 7.802, também conhecida como "Lei do Agrotóxico", de 11 de julho de 1989, que praticamente isenta as indústrias químicas de qualquer responsabilidade quanto ao "uso seguro" dos agrotóxicos no país (SOARES \& PORTO, 2012).

\section{CONCLUSÃO}

A análise cienciomética sobre intoxicação por agrotóxicos permite afirmar que o tema foi pouco abordado pela literatura científica brasileira no período selecionado. Também se conclui que a quase totalidade das pesquisas são realizadas em instituições públicas, principalmente nas regiões Nordeste e Sul. São veiculadas em periódicos exclusivamente do centro-sul brasileiro.

A literatura encontrada relata poucos dados epidemiológicos, como incidência e prevalência, a respeito de intoxicação em trabalhadores e na população de modo geral. Pode-se afirmar que a despeito do fato do Brasil se constituir o maior consumidor mundial de agrotóxicos, poucas pesquisas sobre a saúde do trabalhador rural, em especial sobre a intoxicação por agrotóxicos, são publicadas.

\section{AGRADECIMENTOS}

Ao Centro Universitário de Maringá (UNICESUMAR), ao Conselho Nacional de Desenvolvimento Científico e Tecnológico (CNPq), à Coordenação de Aperfeiçoamento de Pessoal de Nível Superior (CAPES) e Instituto Cesumar de Ciência, Tecnologia e Inovação (ICETI) que contribuíram com esta investigação.

\section{REFERÊNCIAS}

ABREU, P. H. B.; ALONZO, H. G. A. Trabalho rural e riscos à saúde: uma revisão sobre o "uso seguro" de agrotóxicos no Brasil. Ciência e Saúde Coletiva, v. 19, n. 10, p. 4197-4208, 2014. DOI: http://dx.doi.org/10.1590/1413812320141910.09342014

BARBOSA, R. S.; CERQUEIRA, G. S.; LOPES JÚNIOR, H. L.; CARVALHO, S. S.; ALVES, G. C. S. Exposição ocupacional aos agrotóxicos: um estudo bibliográfico. 
Revista Intertox de Toxicologia, Risco Ambiental e Sociedade, v. 7, n. 1, p. 5061, fev. 2014. DOI: http://dx.doi.org/10.22280/revintervol7ed1.166

BRAUN, T., SCHUBERT, A. Scientometric versus socio-economic indicators. Scatter plots for 51 countries, 1978-1980. Scientometrics, v. 13, n. 1, p. 3-9, 1988. Disponível em: http://www.akademiai.com/doi/pdf/10.1007/BF02095758

CAPES. Coordenação de Aperfeiçoamento de Pessoal de Nível Superior. Classificação da Produção Intelectual. Qualis - Periódicos. Disponível em https://sucupira.capes.gov.br/sucupira/public/consultas/coleta/veiculoPublicacaoQual is/listaConsultaGeralPeriodicos.jsf

CARVALHO, C. R. F.; PONCIANO, N. J.; SOUZA, C. L. M. Levantamento dos agrotóxicos e manejo na cultura do tomateiro no município de Cambuci-RJ. Revista Ciência Agrícola, v. 14, n. 1, p. 15-28, 2017. Disponível em: http://www.progep.ufal.br/seer/index.php/revistacienciaagricola/article/view/2327

FERREIRA, M. J. M.; VIANA JUNIOR, M. M. A expansão do agronegócio no semiárido cearense e suas implicações para a saúde, 0 trabalho e 0 ambiente. Interface, v. 20, n. 58, p. 649-660, $2016 . \quad$ DOI: http://dx.doi.org/10.1590/1807-57622015.0029

FERREIRA, T. C.; OLIVEIRA, V. C. Produtos fitossanitários alternativos são inofensivos?. Revista Brasileira de Engenharia de Biossistemas, v. 10, n. 4, p. 416-428, 2016. DOI: http://dx.doi.org/10.18011/bioeng2016v10n4p416-428

GERAGE, J. M.; MEIRA, A. P. G.; SILVA, M. V. Food and nutrition security: pesticide residues in food. Nutrire, v. 42, n. 1, p. 3-10, 2017. DOI: http://dx.doi.org/10.1186/s41110-016-0028-4

MACHADO, D.B.; SANTOS, D.N.; Suicídio no Brasil, de 2000 a 2012. Jornal Brasileiro de Psiquiatria, Rio de Janeiro, v. 64, n. 1, p. 45-54, Mar/ 2015. DOI: http://dx.doi.org/10.1590/0047-2085000000056

MELLO, C. M. de; SILVA, L. F.. Fatores associados à intoxicação por agrotóxicos: estudo transversal com trabalhadores da cafeicultura no sul de Minas Gerais. Epidemiologia e Serviços de Saúde, Brasília, v. 22, n. 4, p.609-619, 2013 DOI: 10.5123/S1679-49742013000400007

OCDE/FAO. Agricultura Brasileira: Perspectivas e Desafios - OCDEFAO 2015. Agricultura Brasileira: Perspectivas e Desafios. 2015. Disponível em: https://www.fao.org.br/download/PA20142015CB.pdf

OPAS/OMS. Manual de vigilância da saúde de populações expostas a agrotóxicos. Brasília: OPAS, 1997. Disponível em: http://bvsms.saude.gov.br/bvs/publicacoes/livro2.pdf

PAPINI, S.; NAKAGAWA, L. E. Current status of rodenticide intoxication in Brazil: a preliminary survey from 2009 to 2011. Brazilian Archives of Biology and 
Technology, v. 57, n. 5, p. 685-688, 2014. DOI: http://dx.doi.org/10.1590/S15168913201402370

ROCHA, T. A. L. C. G.; OLIVEIRA, F. N. Occupational Safety and Health: Vulnerability and perception of risks related to the use of agrochemicals in an irrigated horticulture center of Rio Grande do Norte. Gestão \& Produção, v. 23, n. 3, p. 600-611, 2016. DOI: http://dx.doi.org/10.1590/0104-530x1219-14

SANTANA, V. S.; MOURA, M. C. P.; NOGUEIRA, F. F. Mortalidade por intoxicação ocupacional relacionada a agrotóxicos, 2000-2009, Brasil. Revista Saúde Pública, São Paulo, v. 47, n. 3, p. 598-606, 2013. DOI: http://dx.doi.org/10.1590/S00348910.2013047004306

SCHUBERT, A., GLANZEL, W., BRAUN, T. Scientometric datafiles: a comprehensive set of indicators on 2649 journals and 96 countries in all major science fields and subfields 1981-1985. Scientometrics, n.16, v. 1, p. 3-478, 1989. Disponível em: https://hungary.pure.elsevier.com/en/publications/scientometricdatafiles-a-comprehensive-set-of-indicators-on-2649

SILVA, J. M.; SILVA, E. N.; FARIA, H. P.; PINHEIRO, T. M. M. Agrotóxico e trabalho: uma combinação perigosa para a saúde do trabalhador rural. Ciência e Saúde Coletiva, v. 10, n. 4, p. 891-903, 2005. DOI: http://dx.doi.org/10.1590/S141381232005000400013

SIQUEIRA, S. L.; KRUSE, M. H. L. Agrotóxicos e saúde humana: contribuição dos profissionais do campo da saúde. Revista da Escola de Enfermagem da USP, v. 42, n. 3, p. 584-590, 2008. DOI: http://www.scielo.br/pdf/reeusp/v42n3/v42n3a23.pdf

SOARES, W. L.; PORTO, M. F. S. Uso de agrotóxicos e impactos econômicos sobre a saúde. Revista Saúde Pública, São Paulo, v. 46, n. 2, p. 209-217, 2012. DOI: http://dx.doi.org/10.1590/S0034-89102012005000006.

TAVELLA, L. B.; SILVA, Í. N.; FONTES, L. O.; DIAS, J. R. M.; SILVA, M. I. L. O uso de agrotóxicos na agricultura e suas consequências toxicológicas e ambientais. Agropecuária Científica no Semi-Árido UFCG, Patos, v. 7, n. 2, p. 6-12, 2011. Dispon[ivel em: http://revistas.ufcg.edu.br/acsa/index.php/ACSA/article/view/135

TEIXEIRA, J. R. B.; FERRAZ, J. C. F. C.; NERY, A. A.; CASOTTI, C. A. Intoxicações por agrotóxicos de uso agrícola em estados do Nordeste brasileiro, 19992009. Epidemiologia e Serviços de Saúde, Brasília, v. 23, n. 3, p. 497-508, 2014 . DOI: http://dx.doi.org/10.5123/S1679-49742014000300012

VIEIRA, L. P.; SANTANA, V. T. P.; SUCHARA, E. A. Caracterização de tentativas de suicídios por substâncias exógenas. Caderno de Saúde Coletiva, Rio de Janeiro, v. 23, n. 2, p. 118-123, 2015. DOI: http://dx.doi.org/10.1590/1414-462X201500010074. 